Rabaska

Revue d'ethnologie de l'Amérique française

\title{
Michel Lessard. Le patrimoine québécois inscrit dans la grande histoire
}

\section{Anna-Lou Galassini}

Volume 18, 2020

URI : https://id.erudit.org/iderudit/1072919ar

DOI : https://doi.org/10.7202/1072919ar

Aller au sommaire du numéro

Éditeur(s)

Société québécoise d'ethnologie

ISSN

1703-7433 (imprimé)

1916-7350 (numérique)

Découvrir la revue

Citer ce document

Galassini, A.-L. (2020). Michel Lessard. Le patrimoine québécois inscrit dans la grande histoire. Rabaska, 18, 275-280. https://doi.org/10.7202/1072919ar d'utilisation que vous pouvez consulter en ligne.

https://apropos.erudit.org/fr/usagers/politique-dutilisation/ 


\title{
Michel Lessard. Le patrimoine québécois inscrit dans la grande histoire
}

\author{
AnNA-Lou GalassinI \\ Institut du patrimoine, UQẢM
}

La journée d'étude consacrée à Michel Lessard le 27 février 2020 a été organisée conjointement par l'Institut du patrimoine, le département d'histoire de l'art, les programmes de muséologie de l'UQÀM et la Société québécoise d'ethnologie. Ce fut l'occasion d'entendre des professionnels du patrimoine, anciens collègues de Lessard, mais aussi des jeunes chercheurs. Le but de cette journée consistait à souligner l'apport important de Michel Lessard dans le domaine du patrimoine.

Les séances ont été présidées par divers intervenants : les professeures Lisa Baillargeon (aujourd'hui directrice de l'Institut du patrimoine de l'UQÀM) et Maryse Paquin (représentante du Réseau patrimoine des Universités du Québec), mais aussi par des étudiants : Hubert Alex, Rébecca Lemay-Perrault, Marie-Charlotte Franco, doctorants en muséologie.

Cette journée a débuté par deux allocutions. La première, prononcée par Yves Bergeron, directeur de l'Institut du patrimoine de l'UQÀM, a souligné le rôle important qu'a joué l'UQÀM dans le développement de la discipline et des études en patrimoine. Il a rappelé toutes les figures importantes, dont Michel Lessard, qui ont œuvré dans ce domaine en s'intéressant particulièrement à la culture québécoise, et résumé l'objectif de cette journée : « porter un regard neuf sur la contribution de Michel Lessard ». Dans le même esprit, René Bouchard, rédacteur adjoint de la revue Rabaska, a rappelé l'amour de M. Lessard pour le Québec et ses habitants, qui s'est manifesté par sa pratique intensive du terrain, basée principalement sur la culture matérielle du Québec.

Deux participants ont pris la parole dans la première séance intitulée « Portraits ». René Bouchard est d'abord revenu sur le parcours professionnel de Michel Lessard. Se servant de 1'approche ethnologique comme fil conducteur de sa communication, il a exposé les grands moments de la carrière de Michel Lessard à travers sa vision du patrimoine familial élargi des familles ancêtres Lessard et Poulin de sa lignée, l'enseignement des 
usages quotidiens tiré de l'étude encyclopédique de la culture matérielle du Québec et l'examen minutieux de la photographie ancienne qui en a illustré de larges pans. Engagé comme professeur d'histoire, il se sert des objets contemporains comme tremplin vers ceux du passé pour faire découvrir à ses élèves le passage du temps et l'histoire en marche à travers la culture matérielle du Québec. C'est dans cette perspective qu'il publie la première édition de l'Encyclopédie des antiquités du Québec, ainsi que tous ses autres titres qui connaîtront leur apogée dans sa monumentale Nouvelle Encyclopédie des antiquités du Québec. Il poursuit une carrière florissante dans le domaine du cinéma avec ses célèbres fresques ethnographiques Un pays, un goût, une manière et Les Arts sacrés, et en qualité de professeur en histoire de l'art à l'UQÀm où il fait découvrir toute la richesse documentaire et artistique de la photographie ancienne. Son livre, Québec éternelle, sera son plus grand legs en la matière. Ancien professeur de muséologie à l'UQÀM, collègue et lecteur formé en philosophie, Raymond Montpetit note en tout premier lieu que cette présentation lui pose un défi alors qu'il n'est pas ethnologue. Comme bien d'autres, il confirme que Lessard est alors le principal acteur du patrimoine matériel dans la sphère publique et médiatique, ce qu'il nomme le «Moment Lessard ». Pour ce faire, il met en lumière quatre phases d'une carrière cohérente : premièrement l'encyclopédie, deuxièmement les films documentaires, troisièmement les nouveaux chantiers (histoire de la photographie), et quatrièmement la reprise dans une perspective nouvelle des encyclopédies. Deux fils directeurs suivent son œuvre : l'aspect identitaire et l'appropriation collective. Son intervention a été l'occasion de reconnaître que Lessard est une figure incontournable pour le grand public. Possédant le don de sensibiliser les citoyens au patrimoine, il prône non seulement l'action de l'État, mais aussi l'action citoyenne. Éducateur populaire du patrimoine, grâce à son encyclopédie, il donne à voir à sa manière un musée imaginé, un musée imprimé à l'image d'André Malraux.

La deuxième séance, "Les multiples discours de l'objet », a fait appel à deux intervenants. Paul-Louis Martin, ancien président de la Commission des biens culturels et professeur à l'UQTR, rejoint les propos de Montpetit. Il signale surtout comment la comète Lessard est apparue dans le ciel du patrimoine québécois en opérant d'abord dans la constellation des antiquités - celle des meubles et des objets anciens, mais aussi des maisons et de l'art populaire -, avant de se lancer dans la production de séries documentaires impressionnantes sur les métiers, les traditions et les arts sacrés. Puis, il structure son discours autour de deux évènements particuliers. Le premier concerne la parution, en 1963, du livre de Jean Palardy sur Les Meubles anciens du Canada français, portant sur un patrimoine du XVII ${ }^{\mathrm{e}}$ et $\mathrm{XVIIII} \mathrm{e}^{\mathrm{e}}$ siècles. Le deuxième évènement date de 1965 et il est en lien avec la menace 
de vente à l'étranger de la collection de William. H. Coverdale, répartie pour moitié et dans le Manoir Richelieu et dans l'hôtel Tadoussac ; la dispersion de cette riche collection soulevant des enjeux politiques, il était important de la conserver au pays pour enrichir la collection nationale du Québec. Mais de quel patrimoine parlait-on au juste ? Celui des riches anglais et de la Nouvelle-France? Martin rappelle également deux mesures de conservation, le classement de six arrondissements historiques et la restauration de Place Royale, qui se concentraient également sur la même période. Il conclut que Michel Lessard, prenant le contrepied de ces actions de l'État, a défendu un projet de démocratisation et de réappropriation d'un patrimoine qui fait une place à la production de toutes les époques. Intervenant à son tour, Richard Dubé est revenu sur l'impressionnante carrière de Michel Lessard. En accord avec les précédents conférenciers, il confirme que Lessard est un pédagogue et un expert en communication qui inscrit ses recherches dans le cheminement historique de la société québécoise ; c'est, dit-il, « un homme de parole au verbe haut » qui a su prendre une approche large et englobante qui donne corps à une histoire de notre patrimoine matériel dans toutes ses dimensions, française, britannique, étasunienne et aussi amérindienne. Il évoque sa vision encyclopédique des 400 ans de notre histoire, qui inspireront à Michel Lessard de grandes synthèses sur les objets anciens, le mobilier et les antiquités en général, devenus depuis des références et des repères incontournables signés par un grand maître du patrimoine québécois.

Après ces premières interventions, nous avons eu la chance d'assister à une discussion entre Fernand Dansereau et Michel Lessard, animée par Alexis Lemieux, doctorant à l'UQÀM et spécialiste en cinéma. Dansereau et Lessard reviennent sur les enjeux de la réalisation d'un film ethnographique, notamment par les enquêtes de terrain, dans le cadre des courts métrages réalisés conjointement. De cette collaboration, émerge un apprentissage mutuel : Michel Lessard apprend les techniques du documentaire, du cinéma direct et du langage cinématographique qu'il dit avoir appliquées dans ses propres cours ; de son côté, Fernand Dansereau explique en avoir appris davantage sur la culture matérielle, notamment en se promenant avec Michel dans plusieurs régions et villes sur le territoire. La série documentaire ethnographique Un pays, un goût, une manière, à laquelle les deux hommes ont été associés de très près, constitue un legs important de notre patrimoine cinématographique, malheureusement inconnu par les générations postérieures à 1960 .

Dans le cadre des 50 ans de l'UQÀM, l'Institut du patrimoine produit une série d'entretiens avec des personnalités qui ont marqué le Québec. Cette rencontre avec Michel Lessard était la cinquième de l'année universitaire 2019-2020. Les deux animateurs, Marilie Labonté et Philippe Denis, 
doctorants en muséologie, ont su comprendre l'impact des écrits de Lessard qui se sont révélés incontournables pour leurs premiers emplois dans des institutions muséales! Durant cette rencontre intimiste, Michel Lessard revient sur son parcours et leur confie que son intérêt pour les meubles anciens s'est développé en se promenant. Selon lui, il y a toujours eu un aspect particulier au Québec dans la manière de traiter le patrimoine. Travailler dans ce domaine aura été pour lui travailler pour le Québec et sa fierté.

La séance "Les voies de la médiation » reprend la formule des communications individuelles. Mario Béland, premier intervenant et ancien conservateur au Musée national des beaux-arts du Québec, décrit l'imposante somme des publications de Michel Lessard sur l'histoire de la photographie. Il révèle par ailleurs que Lessard a constitué une importante collection de photographies anciennes de la ville de Québec ; collectionneur, il a acquis des pièces rares et recherchées, dont des portraits de vues urbaines, et a offert sa collection en don au Musée national des beaux-arts du Québec. Jean Simard, vice-président de la Société québécoise d'ethnologie, apporte ensuite un nouvel éclairage en parlant du Musée des religions à Nicolet qui est devenu en 2019 le Musée des cultures du monde. Lessard et Simard ont fait partie tous deux d'un comité d'implantation et participé à la rédaction d'un rapport démontrant la nécessité de créer cette institution muséale. Le rapport Lessard, faisant plus de 400 pages, a constitué la bible de ce nouvel organisme pour ses premières années d'existence. Il a servi de point de départ à la réflexion du comité, en se posant notamment la question : pourquoi Nicolet ? La communication de Simard démontre que le lieu se présente comme l'un des principaux centres de l'histoire de la culture religieuse au Québec et que cette place qu'il a occupée dans cette histoire explique les raisons pour lesquelles il devait s'y implanter.

L'avant-dernière séance, «Actualisation de la recherche » a mis en lumière les interventions de quatre jeunes chercheures en patrimoine. On comprend que la recherche sur le patrimoine est toujours au cœur de l'actualité et en phase avec les préoccupations du temps présent. Contestant la place du patrimoine de la danse contemporaine dans une perspective muséologique, plus précisément dans celle de la réactivation du patrimoine, Julie Anne Côté a noté que la conservation de la danse constitue le plus souvent du «re-enactement»-plutôt qu'une véritable conservation. Aussi a-t-elle soulevé le paradoxe de la danse au musée et le défi que présente la conservation du patrimoine vivant. Laurence Provencher-Saint-Pierre, qui a analysé les modes de collectionnements dans les musées, se questionne sur les pratiques formelles et informelles de collectionnement dans les musées de société. Pour Marie-Josée Gagnon, sa réflexion sur le patrimoine a débuté lors de son stage au Musée des maîtres et artisans de Montréal. Participant à la création 
d'une exposition de mobilier ancien, car elle a une formation d'ébéniste, un banc berçant de nourrice attire son attention. Elle conteste alors l'apport réduit des praticiens au savoir des institutions muséales ; leurs connaissances seraient une véritable plus-value pour les musées dont les collections sont mal documentées. Ainsi, elle focalise son travail sur l'apport des savoirs expérientiels et l'Encyclopédie des antiquités du Québec de Michel Lessard reste pour elle une référence. Marie-Charlotte Franco, dernière intervenante de la séance, indique que sa thèse porte sur l'inclusion des premiers peuples dans les expositions du Musée McCord. Elle montre que le patrimoine matériel et les savoirs expérientiels sont indissociables afin de valoriser les cultures et représentations autochtones. Elle propose donc la décolonisation et l'autochtonisation des musées. Il est important de rappeler que cet aspect contribue à la culture et à la construction des identités culturelles au Québec.

La dernière séance regroupait deux autres communications. Fernand Harvey, de l'InRs, revient sur la signification des notions d'érudition, de collection et de référence identitaire présentes dans l'œuvre de Michel Lessard. Soucieux d'approfondir constamment la connaissance de ses sujets de recherche, tant par la rigueur de leur description que par l'exactitude des faits, Michel Lessard apparaît comme un passionné de l'histoire, de l'objet et de la connaissance. C'est un véritable « érudit dans toute la force du mot». Il démontre l'originalité du travail de terrain de Lessard qui a parcouru toutes les régions du Québec. Il attire notamment l'attention sur les liens que Lessard établit avec les collectionneurs afin de mettre l'objet en relation avec son époque dans une véritable perspective ethnologique. On ne peut s'empêcher de voir en lui un véritable chef d'orchestre qui a su mobiliser des centaines de collaborateurs qui relèvent de l'érudition, des collections et de la recherche scientifique. La dernière communication, signée Gaston Cadrin, rappelle la série d'interventions militantes de Michel Lessard pour la défense du patrimoine naturel et culturel de la grande région de Québec et de Lévis. Ses principales actions militantes ont été menées au fil du temps avec le Groupe d'initiatives et de recherches appliquées au milieu (GIRAM) qui lutte depuis des lustres pour la sauvegarde du patrimoine et qui a derrière lui un actif de quelque 175 conférences de presse dans la défense et l'illustration du patrimoine québécois.

La synthèse présentée par Yves Bergeron au terme de cette journée redisait l'unicité de l'œuvre de Michel Lessard, dont les travaux et les livres ont été inspirants pour le grand public.

Avant de laisser le mot de la fin à Michel Lessard, Jean-Pierre Pichette a fait lecture d'une lettre de Claude Corbo, ancien recteur de l'UQÀM, adressée à Michel Lessard. Son hommage soulignait son importante contribution à la démocratisation des savoirs et aux nouveaux champs de recherche et de 
création, valeurs chères à l'UQÀM. La tenue de cette journée d'étude dans les locaux de l'université prenait alors tout son sens.

Pour clore cette journée, Michel Lessard s'est dit très heureux et touché. Il remercia tous les intervenants et conclut cette journée par un mot tendre pour sa femme, à ses côtés depuis de longues années.

Ce colloque a été l'occasion de redécouvrir la richesse de cette œuvre. Non seulement cette journée a été d'intérêt majeur pour le patrimoine, mais elle fut aussi une occasion pour que d'anciens collègues et amis se retrouvent et mettent en commun leurs expériences. Ce fut aussi un tremplin pour les jeunes chercheurs présents dans l'auditoire venus écouter les interventions de ces figures importantes ayant œuvré pour la conservation du patrimoine québécois.

Les organisateurs planifient l'édition prochaine des actes de cette journée d'étude. 